\title{
Catálisis en el Centro Conjunto de Investigación en Química Sustentable, UAEM-UNAM*
}

\author{
Dora Alicia Solís-Casados, ${ }^{* *}$ Reyna Natividad-Rangel, ${ }^{* * *}$ \\ Rubí Romero Romero, ${ }^{* * *}$ Rosa María Gómez-Espinosa****
}

RESUMEN: Describimos aquí las actividades de investigación en el área de catálisis realizadas por algunas investigadoras de la Facultad de Química de la Universidad Autónoma del Estado de México, adscritas al Centro Conjunto de Investigación en Química Sustentable, UAEM-UNAM. Se incluyen las líneas de investigación que desarrollan, las contribuciones que han generado y los logros en este campo obtenidos en los últimos años. También se presenta información sobre la infraestructura con que se cuenta en el CCIQS y en la Facultad de Química para llevar a cabo investigación sobre catálisis; la importancia que tienen los trabajos de colaboración nacional e internacional, así como, la formación de recursos humanos altamente especializados en esta área. Por último, presentamos las perspectivas de investigación y desarrollo planteadas para el campo de la catálisis.

PALABRAS CLAVE: Catálisis, nanocatálisis, fotocatálisis, energías limpias, medio ambiente, biocombustibles.

ABSTRACT: This manuscript describes the research activities in the area of catalysis that are carried out by researchers from the Faculty of Chemistry of the Autonomous University of the State of Mexico and are affiliated to the Joint Center for Research in Sustainable Chemistry (Spanish acronym: CCIQS, UAEM-UNAM). It includes the lines of research that they develop, the contributions they have generated and the achievements in this field in the last years. It also presents information on the infrastructure that is available in the CCIQs and in the Faculty of Chemistry to carry out research on catalysis. It is also included the importan-

Recibido: 13 de febrero de 2017. Aceptado: 27 de marzo de 2017.

* Deseamos expresar nuestro más profundo agradecimiento al conACyT por los apoyos brindados para no solamente realizar investigación en el área de catálisis sino también por el apoyo recibido para la formación de recursos humanos altamente especializados y por el SNI. En el mismo contexto, extendemos el agradecimiento a PRODEP, UAEM y UNAM. Por otro lado, también queremos agradecer a nuestros compañeros del CCIQS UAEM-UNAM, quienes nos han apoyado en las diferentes cuestiones técnicas implícitas en nuestros proyectos: M.C. Alejandra Núñez, M.C. Lizbeth Triana, Dr. C. Uvaldo Hernández, Dra. Melina Tapia y Lic. Citlalit Martínez Soto. Finalmente, a nuestros estudiantes y pares que han tenido confianza en nuestros proyectos y han contribuido de manera importante a la calidad de nuestros productos académicos y científicos.

** Cuerpo Académico en Ciencia de Materiales, Centro Conjunto de Investigación en Química Sustentable (CCIQS), UAEM-UNAM, Facultad de Química, Universidad Autónoma del Estado de México. Correspondencia: (solis_casados@yahoo.com.mx).

*** Cuerpo Académico en Ingeniería Química, (CCIQS), UAEM-UNAM. Correspondencia: (reynanr@gmail.com).

**** Cuerpo Académico en Química de Coordinación, (CCIQS), UAEM-UNAM. Correspondencia: (rosamarigo@gmail.com). 
ce of national and international collaboration, as well as the training of highly specialized human resources in this area. We present the perspectives of research and development that we propose in the field of catalysis.

KEYWORDS: Catalysis, nanocatalysis, photocatalysis, clean energy, environment, biofuels.

\section{Introducción}

La Facultad de Química de la Universidad Autónoma del Estado de México (UAEM) se creó en 1970 con el nombre de Instituto en Ciencias Químicas impartiendo las primeras clases en las instalaciones de la Facultad de Medicina de la misma universidad, posteriormente en el año de 1975, se llamó Escuela de Ciencias Químicas, inaugurándose el actual edificio ubicado en Paseo Colón Esquina con Paseo Tollocan en la Ciudad de Toluca del Estado de México. En esos años solo se impartían las carreras de químico y químico farmacéutico biólogo, creándose en el año de 1978 la carrera de químico en alimentos, siendo la docencia la principal actividad de la Escuela en Ciencias Químicas en esa época, la enseñanza en el posgrado comenzó en 1985 con la maestría en ecología, dando inicio a las actividades de investigación en el área de química en este espacio universitario. Estas actividades de investigación se han visto fortalecidas con el transcurso del tiempo y las contrataciones de personal como profesores de tiempo completo de la UAEM, de manera adicional estas actividades de investigación se han visto incrementadas con la firma de un convenio entre la Facultad de Química de la UAEM y el Instituto de Química de la UNAM, el 24 de mayo de 2007, haciendo posible la creación de un Centro Conjunto de Investigación en Química Sustentable (CCIQS), en el convenio se menciona que en el CCIQS UAEM-UNAM <http://www.cciqs.unam.mx > debe haber: "Una relación donde ambas universidades compartan riesgos, afronten problemas-retos y colaboren en actividades académicas de docencia e investigación, todo lo anterior de manera conjunta. En este modelo se concibe a la UNAM como una institución de apoyo y no de competencia con la Facultad de Química de la UAEM durante un tiempo determinado en el convenio de colaboración". El día 9 de septiembre de 2008 se inauguró el CCIQS UAEM-UNAM (figura 1), en la actualidad se encuentran adscritos a este centro 18 profesores de tiempo completo pertenecientes a la UAEM y 8 investigadores pertenecientes a la UNAM. De la misma manera, fueron integrándose 7 técnicos académicos por la UNAM en apoyo al manejo de infraestructura especializada. Se pretende que este personal académico lleve a cabo actividades de investigación enfocadas principalmente a la química sustentable, donde destacan la ciencia de materiales, la nanociencia, la nanotecnología, la electroquímica, química del estado sólido, fisicoquímica teórica, remediación de agua y los procesos de oxidación avanzada. 


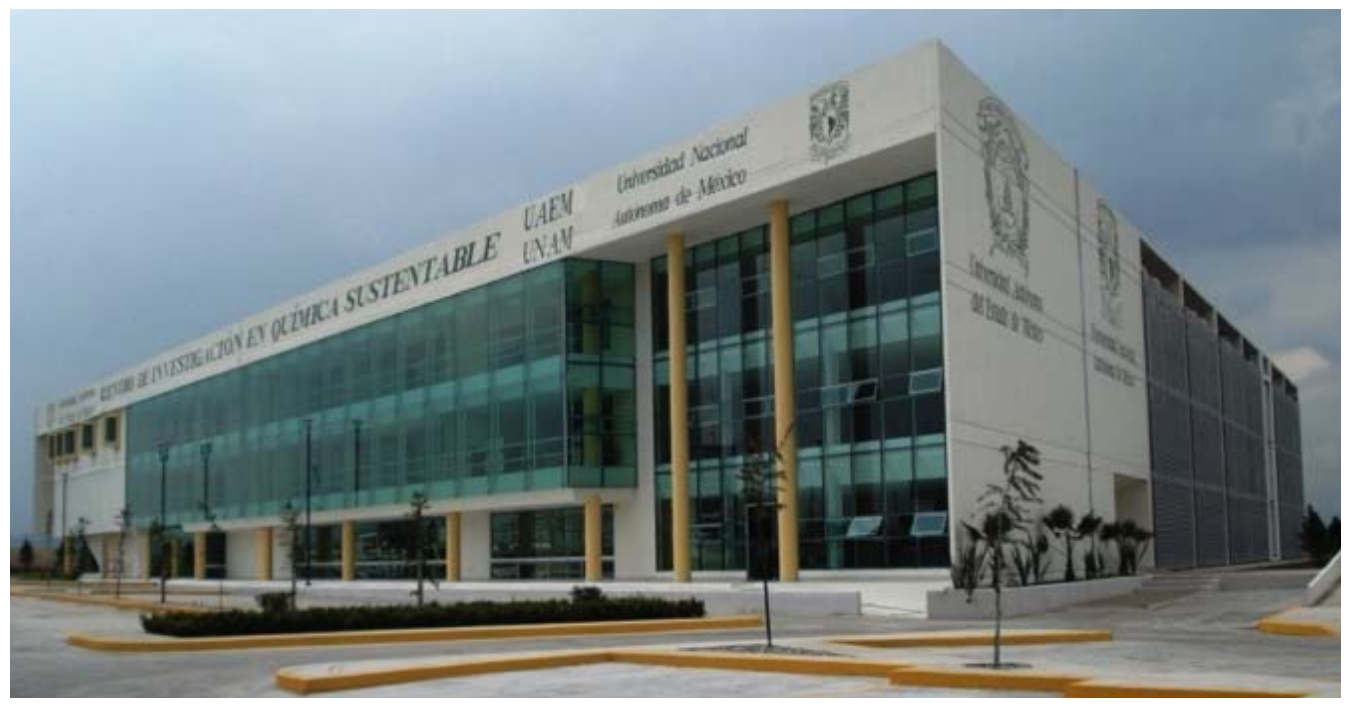

FIGURA 1. Fachada del CCIQS, UAEM-UNAM.

\section{Líneas de investigación, desarrollo e innovación relacionadas con la catálisis}

El personal de investigación se ha agrupado en torno a 6 líneas principales, fisicoquímica, ingeniería química, química de materiales, química inorgánica, química ambiental y química orgánica; algunas investigadoras han desarrollado, en las líneas de ingeniería química, química de materiales y química inorgánica, su investigación enfocada en el área de catálisis, principalmente en reacciones de catálisis heterogénea, y en algunos casos se ha desarrollado catálisis homogénea. Las líneas de investigación que se desarrollan e impactan el área de catálisis son la de síntesis caracterización y desarrollo de materiales con aplicaciones catalíticas, mineralización de compuestos orgánicos mediante fotocatálisis heterogénea, foto-oxidación selectiva de compuestos orgánicos (glicerol, alcoholes), foto-reducción catalizada de $\mathrm{CO}_{2}$, fotofenton y ozonación catalizada, la modificación de catalizadores homogéneos con ligantes estibinicos con aplicación en la reacción de amidocarbonilación, heterogenización de un catalizador homogéneo.

\section{Investigación y desarrollo}

En la facultad de Química de la UAEM se ha desarrollado investigación usando catalizadores en reacciones principalmente homogéneas por algunos profesores del área de química orgánica, investigaciones fortalecidas con la llegada de la Dra. Rosa María Gómez Espinosa en el año 2004, quien se incorpora a tra- 
vés del programa de repatriación del CONACyT. La investigación en catálisis heterogénea dio inicio en el año 2003 con la Dra. Rubí Romero Romero, quien se reincorporó tras terminar los estudios de doctorado, incorporándose a la Facultad de Química de la UAEM; en 2005, la Dra. Reyna Natividad Rangel y en 2006 la Dra. Dora Alicia Solís Casados, esta última a través del programa de Retención del Consejo Nacional de Ciencia y Tecnología (cONACyT). Estas investigadoras, a partir de 2003 a la fecha, han desarrollado proyectos de investigación en el área de catálisis heterogénea principalmente en el desarrollo de materiales catalíticos, fotocatalíticos, caracterización física y química de los mismos, así como el estudio de los parámetros cinéticos y la evaluación de la actividad catalítica, optimizando en algunos casos el sistema de reacción.

En orden cronológico, la Dra. Rosa María Gómez Espinosa, perteneciente al Cuerpo Académico de Química de Coordinación, ha dedicado estos últimos 12 años de investigación científica a desarrollar catalizadores soportados en matrices poliméricas, obteniendo como principal logro la modificación de catalizadores homogéneos con ligantes estibínicos con aplicación en la reacción de amidocarbonilación y la heterogenización de un catalizador homogéneo. La Dra. Gómez ha desarrollado numerosos proyectos (PRODEP, CONACYT, SIyEA) que inciden en el área catalítica, lo cual se ve reflejado en los numerosos artículos publicados entre los que destacan los publicados en una revista alemana en el año 2000 [1] y en el Journal of Molecular Catalysis en los años 2001, 2004 y 2008 [2-4]; adicionalmente, publicó un capítulo titulado "Catalizadores soportados" en el libro Tópicos en materiales. En el tema de formación de recursos humanos, 19 estudiantes de licenciatura pertenecientes a la Facultad de Química se han recibido, y en el posgrado se han graduado 10 altamente especializados en la maestría, y 5 doctores.

La Dra. Natividad es miembro del Cuerpo Académico Consolidado de Ingeniería Química y adscrita al cCIQs, ha invertido gran esfuerzo en cultivar las líneas de investigación como fotooxidación selectiva de compuestos orgánicos, fotorreducción catalizada de $\mathrm{CO}_{2}$, mineralización de compuestos orgánicos mediante fotocatálisis heterogénea, fotofenton y ozonación catalizada [5-8]. Ha dirigido numerosos proyectos de investigación financiados por la UAEM, PROMEP y CONACYT, entre otros, con lo cual ha adquirido la infraestructura necesaria para llevar a cabo las actividades de investigación en las líneas mencionadas, fortaleciendo el equipamiento básico del laboratorio con la adquisición y puesta en operación de tres fotorreactores de $100 \mathrm{~mL}$, dos fotorreactores de 1L, una columna de burbujeo de 14L y una columna de burbujeo de 4L (figuras 2-3), un analizador de carbono orgánico total (COT) Shimadzu. Esta infraestructura le ha permitido formar recursos humanos especializados en el área de catálisis, trabajo reflejado en la dirección de 33 tesis: 16 de licenciatura en ingeniería química, 14 de maestría y 13 de doctorado en ciencias químicas; la Dra. Natividad ha publicado a la fecha 50 artículos en revistas indizadas, 4 capítulos de libro, y cuenta con dos solicitudes de patente en proceso. Es importante resaltar que la Dra. Natividad ha fortalecido las colaboraciones con in- 


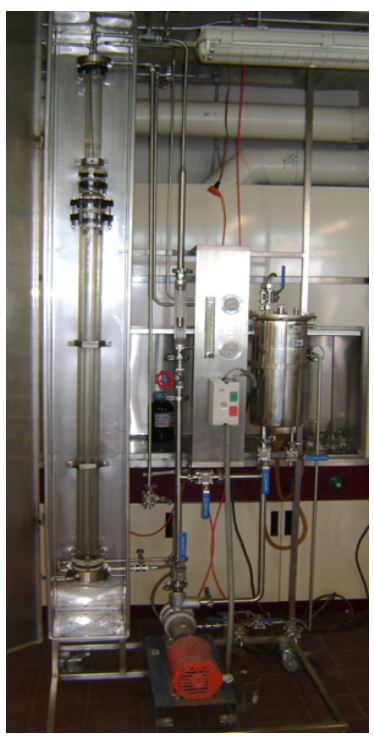

FIGURA 2. Columna de burbujeo: reactor fotocatalítico $\mathrm{y} / \mathrm{o}$ electroquímico. vestigadores adscritos a instituciones dentro de la república mexicana citándose la Universidad Michoacana de San Nicolás de Hidalgo, el Instituto Mexicano del Petróleo, la Universidad Nacional Autónoma de México y ha trabajado fuertemente en otras colaboraciones con instituciones en el extranjero destacándose la University of Birmingham y University of Loughborough del Reino Unido, así como la Universidad Politécnica de Valencia, España. Es importante resaltar que la Dra. Natividad ha recibido varias distinciones nacionales como internacionales por su destacada labor en el área de catálisis heterogénea. En 2015, fungió como presidente del XIV Congreso Mexicano y V Congreso Internacional de Catálisis. Actualmente, es vocal del consejo directivo de la Academia de Catálisis.

La Dra. Rubí Romero pertenece al Cuerpo Académico de Ingeniería Química y ha fortalecido la línea de investigación de catálisis e ingeniería

FIGURA3. a) Reactor STR con condensador para la producción de biodiesel mediante la transesterificación de aceites vegetales catalizada heterogéneamente; b) fotorreactor STR para la evaluación catalítica de fotocatalizadores.
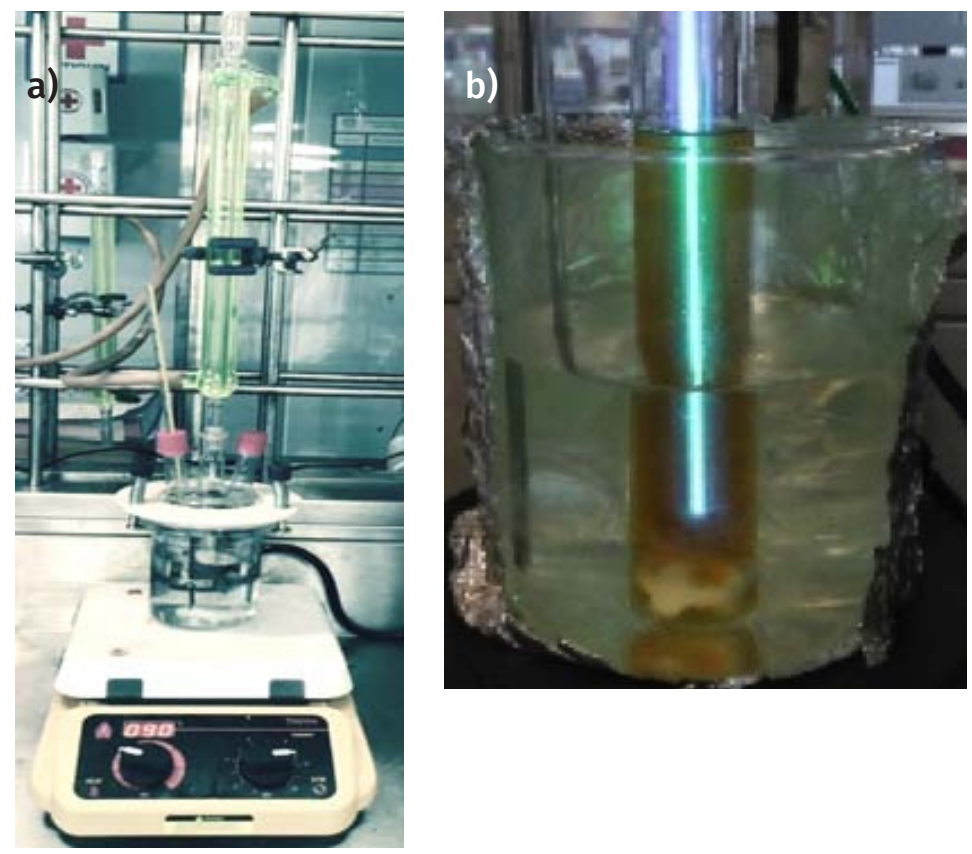
de las reacciones químicas en el cCIQS. Ha dirigido al menos diez proyectos de investigación financiados por instituciones como la UAEM, SEP- PROMEP, CONACyT-ciencia básica y uno de cooperación internacional financiado por la Junta de Comunidades de Castilla-La Mancha y Fondos FEDER. 2007-2009. Es importante resaltar que los recursos recibidos han incidido de manera directa en fortalecer las actividades de catálisis, debido a que han permitido adquirir infraestructura como lo son los reactores tipo Batch y algunos reactores fotocatalíticos en escala laboratorio. En el marco de estos proyectos se han formado recursos humanos especializados, graduándose 15 alumnos de nivel licenciatura, 7 alumnos de la maestría en ciencias químicas y 3 alumnos de doctorado. Derivado del trabajo de investigación la Dra. Romero ha publicado más de 30 artículos en revistas indizadas, y recibido distinciones tanto nacionales como internacionales por su destacada labor en el área catalítica [9-16]. En 2015, participó en el comité organizador del XIV Congreso Mexicano y V Congreso Internacional de Catálisis.

La Dra. Dora Alicia Solís Casados ha cultivado la línea de investigación en catálisis heterogénea, llevando a cabo investigación en la síntesis, caracterización y desarrollo de materiales porosos, nanoestructuras, nanopartículas y de manera adicional ha trabajado en la obtención de materiales a base de óxidos metálicos en forma de película delgada. De estos materiales, ha evaluado su actividad catalítica en reacciones fotocatalíticas y en algunos casos en reacciones de hidrodesulfuración selectiva. Ha dedicado gran parte de sus actividades de investigación al desarrollo y caracterización de películas delgadas de óxidos de metales de transición obtenidos a partir de soluciones de sol-gel y depositadas usando el depósito por giro (spin coating), materiales modificados sensibles a la luz solar y que ha evaluado en la degradación fotocatalítica de compuestos orgánicos en aguas residuales. Como resultado de sus actividades de investigación, ha publicado más de 45 artículos en revistas indizadas [17-21]; ha formado recursos humanos altamente especializados reflejados en las tesis dirigidas, contando con 3 de licenciatura en las carreras de química e ingeniería química, 5 de maestría en ciencia de materiales y 1 de maestría en ciencias ambientales, así como 7 de doctorado. Es importante hacer notar que la Dra. Solís ha recibido distinciones nacionales y algunas internacionales por su destacada labor en el área de catálisis heterogénea. En 2015, participó en el comité organizador del XIV Congreso Mexicano y V Congreso Internacional de Catálisis. Hoy en día, es secretaria del Consejo Directivo de la Academia de Catálisis, A.C.

\section{Infraestructura}

Con el financiamiento otorgado por diversas instituciones como la misma UAEM, SEP-PROMEP y CONACYT, se ha logrado obtener recursos empleados en equipamiento para fortalecer la infraestructura disponible y con ello la obtención de mejores resultados en el área de catálisis. Se ha adquirido: un es- 
Mundo Nano | CATÁLISIS EN MÉXICo | www.mundonano.unam.mx

10(18), enero-junio 2017 | DOI: 10.22201/ceiich.24485691e.2017.18.60107

pectrofotómetro UV-vis lambda 35 de Perkin Elmer (figura 4a), un simulador solar marca Science Tech (figura 4b), un reactor de fotoquímica Q-200 Marca Prendo (figura 5a), autoclave (figura 5b), microscopio óptico digital, así como lámparas ultravioleta, rotavapor (figura 6 a), horno para calcinar (figura 6 b), equipo Soxleth, pHmetro, turbidimetro, microcentrífuga, equipo de fisisorción CHEMBET 3000 y un equipo de depósito por giro (figura $7 \mathrm{~b}$ ), entre otro instrumental y equipo de laboratorio menor que le han servido de apoyo para dar seguimiento al desempeño de las formulaciones catalíticas preparadas.
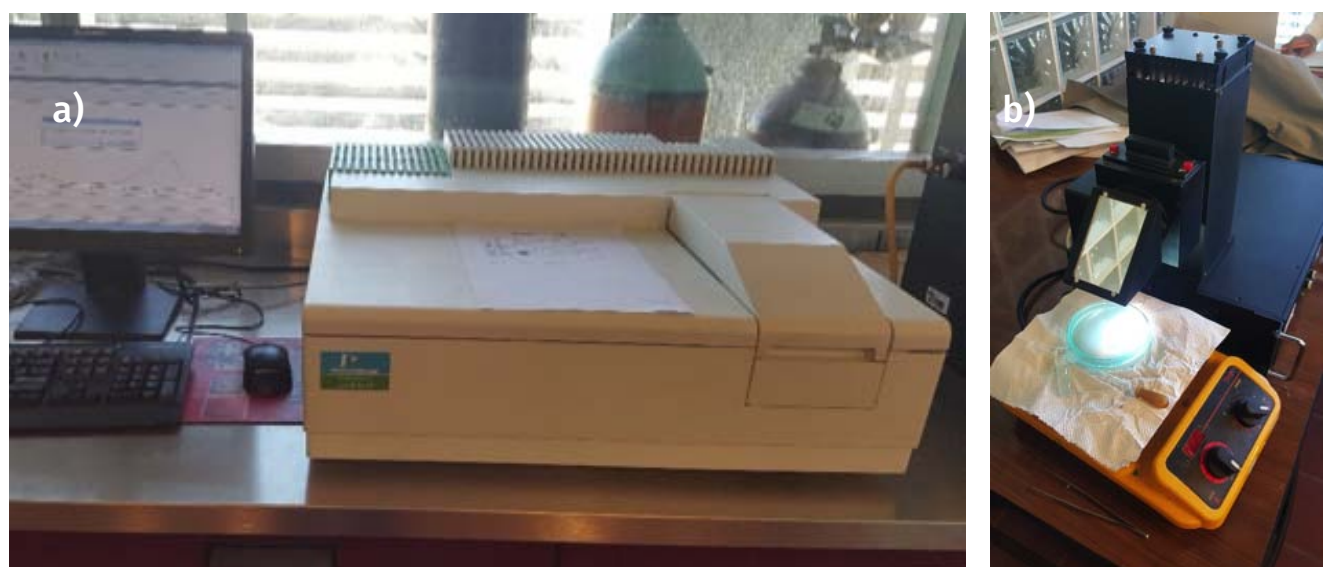

FIGURA 4. a) Espectrofotómetro Lambda 35 de Perkin Elmer; b) simulador solar Science Tech.

FIGURA 5. a) Reactor de fotoquímica con lámpara UV; b) reactor de alta presión marca Prendo.
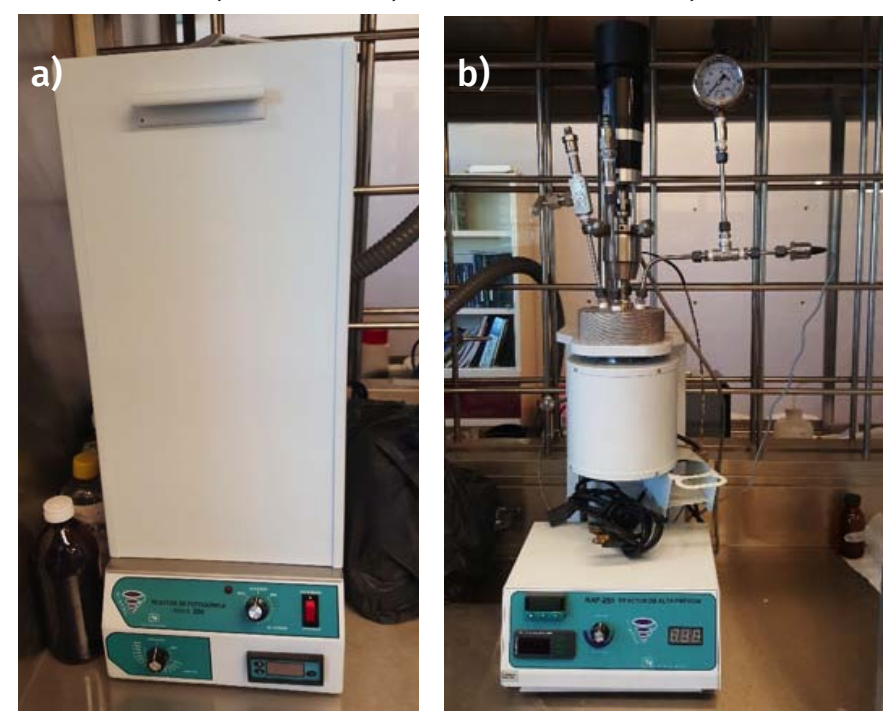
infrarrojo (IR-ATR), un equipo de resonancia magnética nuclear (NMR), un equipo de espectrometría fotoelectrónica de rayos X (XPS). El cCIQS también cuenta con una sección en la cual se dispone de equipos de cromatografía que apoyan la identificación de diferentes subproductos de reacción muy útiles en el área de catálisis. Recientemente se adquirieron tres cromatógrafos mediante el proyecto "Apoyo al fortalecimiento de la infraestructura del área de catálisis e ingeniería de reacciones del CCIQS UAEM-UNAM" teniendo como marco la convocatoria de CONACyT de apoyo a la infraestructura 2016 bajo la responsabilidad de la Dra. Natividad y con la colaboración, entre otros, de la Dra. Solís, las doctoras Gómez y Romero. Los cromatógrafos así adquiridos son un UHPLC con detector de arreglo de diodos y con índice de refracción, un cromatógrafo de gases con detectores de ionización de flama (FID) y de conductividad térmica (TCD) y un cromatógrafo de iones. Estos equipos son excelentes para detectar y cuantificar compuestos de interés de los proyectos realizados por las autoras de este trabajo como son la fotoxidación selectiva (de glicerol) y total (de compuestos fenólicos y contaminantes emergentes) y de la fotorreducción de $\mathrm{CO}_{2}$, producción de alcoholes, transesterificación de aceites vegetales (biodiesel) y remoción de iones.

\section{Actividades de vinculación en el área de catálisis}

La vinculación se lleva a cabo con otros investigadores en instituciones nacionales e internacionales, siendo importante resaltar el fortalecimiento de las colaboraciones con investigadores adscritos a instituciones dentro de la república mexicana citándose la Universidad Nacional Autónoma de México (UNAM), Universidad Autónoma Metropolitana (UAM), Instituto Politécnico Nacional (IPN), Instituto Mexicano del Petróleo (IMP), Tecnológico de Estudios Superiores de Jocotitlan (TESJO), Universidad Tecnológica de Zinacantepec (UTZIN), Instituto Tecnológico de Toluca (ITT), Instituto Tecnológico de Estudios Superiores de Monterrey (ITESM), Instituto Nacional de Investigaciones Nucleares (ININ), Universidad Michoacana de San Nicolás de Hidalgo (UMSNH), Universidad Autónoma de Querétaro (UAQ) y otras vinculaciones de carácter internacional como la Universidad de Málaga en España (UMA), Universidad Tecnológica de Valencia en España, Universidad de Castilla-La Mancha en España (UCLM), University of Birmingham, University of Loughborough del Reino Unido y Thompson Rivers University de la Columbia Británica en Canadá. Como resultado de estas colaboraciones se han publicado artículos en revistas indizadas y en algunos casos los estudiantes han realizado estancias de investigación en alguna de estas instituciones, lo cual ha robustecido los proyectos de investigación en el marco de los cuales se desarrollan sus trabajos de tesis y los posgrados a los que pertenecen dichos estudiantes (Ciencias Químicas <http://web.uaemex.mx/fquimica/posgrados/ $\mathrm{CQ} />$, y Ciencia de Materiales <http://web.uaemex.mx/fquimica/posgrados/ $\mathrm{CM} />)$. 


\section{Referencias}

[1]. Gómez R. M., Sharma P., Arias J. L., Pérez-Flores J., Velasco L., Cabrera A. (2001). Amidocarbonylation of cyclohexene and 1-pentene with $\mathrm{CO}_{2}(\mathrm{CO})_{8}$ modified with triarylstibines in very mild conditions. Journal of Molecular Catalysis A: Chemical, 170 (1-2): 271-274, mayo 14. issn: 1381-1169.

[2]. Cabrera A., Sharma P., Arias J. L. et al. (2004). Amidocarbonylation of alkenes at very low pressures with a $\mathrm{CO}_{2}(\mathrm{CO})_{8} / \mathrm{SbR}_{3}$ system: Two easy routes to reach nacetyl-alpha-aminoacids. Journal of Molecular Catalysis A: Chemical, 212 (1-2): 19-23. doi:10.1016/j.molcata. 2003.11.009. ISSN: 1381-1169.

[3]. Gómez R.M., Cabrera A., Velázquez C.G. (2007). Synthesis of stibine ( $\operatorname{sbphrr}_{2} r_{1}$ and their use as ligand in the amidocarbonylation of alkenes with $\mathrm{CO}_{2}(\mathrm{CO})_{8}$ as precursor. Journal of Molecular Catalysis A-Chemical, 274 (1-2): 65-67 2007. doi:10.1016/j.molcata.04.018. ISSN: 1381-1169.

[4]. Salinas-Olvera, J.; Gómez, R. M.; Cortés-Guzmán, F. (2008). Structural evolution: Mechanism of olefine insertion in hydroformylation reaction. Journal of Physical Chemistry, 112. (13), 2906-2912, 3 abril. doi: 10.1021/jp711200n. ISSN 1089-5639.

[5]. Hurtado L., Solís-Casados D., Escobar-Alarcón L., Romero R., Natividad R. (2016). Multiphase photo-capillary reactors coated with $\mathrm{TiO}_{2}$ films: Preparation, characterization and photocatalytic performance. Chemical Engineering Journal, 304:39-47.

[6]. L. Hurtado, R. Natividad, H. García. (2016). Photocatalytic activity of $\mathrm{Cu}_{2} \mathrm{O}$ supported on multi layers graphene for $\mathrm{CO}_{2}$ reduction by water under batch and continuous flow. Catalysis Communications, 2016, 84:30-35.

[7]. L. Hurtado, R. Natividad, E. Torres-García, J. Farías, G. Li Puma. (2015). Correlating the photocatalytic activity and the optical properties of $\mathrm{LiVMoO}_{6}$ photocatalyst under the UV and the visible region of the solar radiation spectrum. Chemical Engineering Journal, 262, 1284-1291.

[8]. E. Martín del Campo, J. S. Valente, T. Pavón, R. Romero, A. Mantilla y R. Natividad. (2011). 4-chlorophenol oxidation photocatalyzed y a calcined Mg-Al-Zn layered double hydroxide in a concurrent downflow bubble column. Industrial Engineering Chemistry Research, 50 (20), 11544-11552.

[9]. Muciño, G.E.G., Romero, R., García-Orozco, I., Serrano, A.R., Jiménez, R.B., Natividad. (2016). R. Deactivation study of $\mathrm{K}_{2} \mathrm{O} / \mathrm{NaX}$ and $\mathrm{Na}_{2} \mathrm{O} / \mathrm{NaX}$ catalysts for biodiesel production. Catalysis Today, 271, 220-226.

[10]. Muciño, G.G., Romero, R., Ramírez, A., Martínez, S.L., Baeza-Jiménez, R., Natividad, R. (2014). Biodiesel production from used cooking oil and sea sand as heterogeneous catalyst. Fuel, 138, 143-148.

[11]. Peña, R., Romero, R., Martínez, S.L., Natividad, R., Ramírez, A. (2013). Characterization of $\mathrm{KNO}_{3} / \mathrm{NaX}$ catalyst for sunflower oil transesterification. Fuel, 110, 63-69.

[12]. Luz Martínez, S., Romero, R., López, J.C., Romero, A., Sánchez-Mendieta, V., Natividad, R. (2011). Preparation and characterization of $\mathrm{CaO}$ nanoparticles/ 
$\mathrm{NaX}$ zeolite catalysts for the transesterification of sunflower oil. Industrial and Engineering Chemistry Research, 50, (5) 2665-2670.

[13]. Peña, R., Romero, R., Martínez, S.L., Ramos, M.J., Martínez, A., Natividad, R. (2008). Transesterification of castor oil: Effect of catalyst and co-solvent. Industrial and Engineering Chemistry Research, 48 (3) 1186-1189.

[14]. Ramos, M.J., Casas, A., Rodríguez, L., Romero, R.,Pérez, A. (2008). Transesterification of sunflower oil over zeolites using different metal loading: A case of leaching and agglomeration studies. Applied Catalysis A: Gen., 346 (1-2) 79-85.

[15]. Valverde, J.L., Romero, A., Romero, R., García, P.B., Sánchez, M.L., Asencio, I. (2005). Preparation and characterization of Fe-PILCS. Influence of the synthesis parameters. Clays and Clay Minerals, 53 (6) 613-621.

[16]. Dorado, F., Romero, R., Cañizares, P. (2002). Hydroisomerization of n-butane over $\mathrm{Pd} / \mathrm{HZSM}-5$ and $\mathrm{Pd} / \mathrm{H} \beta$ with and without binder. Applied Catalysis A: Gen., 236 (1-2), 235-243.

[17]. D. A. Solís-Casados, L. Escobar-Alarcón, A. Infantes-Molina, T. Klimova, Lizbeth Serrato-García, Enrique Rodríguez-Castellón, Susana Hernández-López y Alejandro Dorazco-González. (2017). Synthesis and characterization of ag-modified $\mathrm{v}_{2} \mathrm{O}_{5}$ photocatalytic materials. Journal of Chemistry, ID 5849103, doi. ORG/10.1155/ 2017/ 5849103.

[18]. D.A. Solís-Casados, L. Escobar-Alarcón, L.M. Gómez-Oliván, E. Haro-Poniatowski, T. Klimova. (2017). Photodegradation of pharmaceutical drugs using Sn-modified $\mathrm{TiO}_{2}$ powders under visible light irradiation. Fuel, 198, 3-10.

[19]. L. Escobar-Alarcón, D.A. Solís-Casados, F. González-Zavala, S. Romero, M. Fernandez y E. Haro-Poniatowski. (2017). Preparation of nanostructured Bi-modified $\mathrm{TiO}_{2}$ thin films by crossed-beam laser ablation plasmas. IOP Conf. Series: Journal of Physics: Conf. Series, 792 012006, Dor: 10.1088/17426596/792/1/ 01200.

[20]. D. A. Solís-Casados, L. Escobar-Alarcón, T. Klimova, E. Rodriguez-Castellón, J.A. Cecilia, C. Morales-Ramírez, C. (2016). Catalytic performance of CoMo/ $\mathrm{Al}_{2} \mathrm{O}_{3}-\mathrm{MgO}-\mathrm{Li}(\mathrm{x})$ formulations in DBT hydrodesulfurization. Catalysis Today, 271, 35-44.

[21]. L. Escobar-Alarcón, D.A. Solís-Casados, M. Fernández y F. Valencia. (2013). Malachite green degradation in simulated wastewater using $\mathrm{Ni}_{\mathrm{x}}: \mathrm{TiO}_{2}$ thin films. Fuel, 110, 2013, 17-22. 